\title{
Sach-Register für Band XLIV
}

Die fctt gedruckten Zahlen bedeuteá Original-Artikel. Bz. = Buchanzeigen. P. = Personalien. A.

Aderhautablö sung, abnorm lange

persístierende. 182. \Aderhaatmelanom. 326. Aderhautsarkom ohne Abhebung

der Netzhaut. 219. Akkommodation, Einfluß der auf

die Verengerung der Pupille. 329. Akkommodationsbreite, Einñuß

der Heterophorie aùf die. 98. Akkommodationsfähigkeit, Ein-

$11 \alpha ß$ des Krieges auf die. 140. Amblyopieu, Häufung toxischer

gegenüber der Zeit voii· dem Kriege.

$38,105,198$.

- Wirkungen des Lichtes bei toxi-

schen. 325. Angoid streaks. 217, Anomalien, angeborene des Auges.

90 ff. A rgyll - Ro ber tsonsches Phä-

nomen, umgekehrtes. 331. Auge, Verlust eines vor dem Unfall

erbiindeten. 336. Augenbec herspalte. 316. Aagendruck, Einfluß der Pupillen-

weite aaf den bei Glaukom. 325. Augenhintergrund, Anomalien

des. 92. Augenhöhlen- Nebenhöhl en -

fistelii, plastischer Verschl-iß

von. 342. Augenlider, Verletzungen der. 101.

- Behandlung frischer Verletzungen

der. 343.

- $\quad$ beiderseitige Geschwulst der, kom-

biniert mit tieschwulstbildung der OberПppe. 17('». Augenverletzungen, Mechanik der. 321.

B.

Bäder, Sehstörungen im Anschluß

an heiße. 342. Ba dkon j unctivitis. 13.

Bändertrübung, Haabsche. 242. B i n d e li a u t, Con j uncti va neuro-aller-gica,. 224. -

- $\quad$ eigentümiiche Form koujunktivaler

Geschwürsbildung. 215.

- $\quad$ Tuberkulide der. 222.Binokularlampe für endoskp-

pische Zwecke. 326.

Blindheit, hysterische. 331.

Blut, UntersucKungeu des bei Glaukom. 306, H07.

Blutverlust, Selistörungen Each. 116.

Bogengang, Zusammenhang zwi-schen der Kichtung des Nystagmus mid dem Peize in einem. 99.

B r e c h u n g s v e r m e h r u n g in der Dämmerung. 98.

Brillengläser für Presbj^ope und Amblyope. 322.

C. 
Conjunctivitis, Bad-C. 13. Conjunctivitis gonorrhoica, Behandlung der. 99.

- $\quad$ parenterale Milchinjektionen bei.

$145,178,184,216$.

D.

Dämm erung, Brechungsvermehrung

in der. 98. Diabetes und Trauma. 113. Dilatator iridis. 304. Dystrophia epithelialis, Pig-

mentierung der Hornhautliinter-

fläche bei. 247.

E.

Einäugige, Puchtungslokalisation

und Angewöhnung der. 311. Elektrischer Schlag, doppel-

seitige Katarakt mit Quellungs-

glaukom nach. 324. Elliotsche Trepanation. 309,

329.

Sach-Register zi

Embryotoxon corneae posterior.

324. Encephalitis lethargica, Augen-

symptome bei. 232. Enukleation, Operatione $\alpha$ zur Ver-

besserung der Kosmetik nach. 333 Eosinophilie, lokale. 314. Epicanthus, Morpbogenesedes. 95.

Erythema nodosum, Ringkooten

des Ziliarkörpers, bei. 231. Eukupin, Schädigungen des Auges

durch. 313. E v o 1 u t i o n s s tö r u.ng, universelle

qualitative. 95. Exophthalmus, iatermittierender.

153.

F.

Earbensinnprüfung mit de $\alpha$ HelmboldschenFarbenscbeiben 330.

Earbensinnstörungen, Apparat zur Untersuchung von. 327.

Flüssigkeitswechsel im Auge. 305.

Fragilitas ossiumund blaue Skle-rae. 100.

G.

Geschlechtsgebundene Krank-heiten, Vererbung der. 303.

Gesichtsfeld, Untersuchung des peripheren. 322.

- $\quad \Lambda$ Vettstreit der. 98. Gewebskulturen in vitro nach

Carrels Methode. 325. Glaskörperverlust, Verhütung des

bei Augenoperationen. 101. Glaukom, Mechanik des 307.

- $\quad$ Einfluß der Pupillenweite auf den

Augendruck bei. 325.

Blut- und Kammerwasserunterr suchungen bei. 306, 307.

und hochgradige Myopie 308.

nach Papiilitis. 308.

doppelseitige Katarakt mit Quel-

lungsglaukom nach eléktrischem

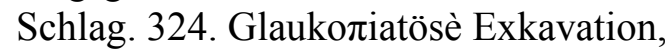

Rückgang der. 225. Glioma retinae. 327. Grippe, als Ursache reflektorischer j Pupillenstarre. 44.

H.

Hemianopische Pupillenreak- i 
tion. 315. Hemianopsie, binasale. 100. Herpes zoster. 74, 103.

- $\quad$ corneae. 313. Heterophorie, Einfluß der auf die

Akkommodationsbreite. 98.Heterotopie des öehnerven und derMakula. 92..'

Band XLIV. 353

Hornhaut, vertikal-ovale. 102.

- $\quad$ Regeneration einfacher und perfo-

rierender Defekte der. 326.

feder. 100

Verletzune: der durch eine Schreib-

- Behandlung entzündlicher Er-krankungen der mit ultraviolettemLicht. 1, 332.

HornhauthinterfГãche, Pigmen-

tierung der bei Dystrophia epi-

thelialis. 247. Hornhauttrübungen, angeborene $1 / 8$

92, 93, 220, b27. Hydrophthalmus, vererbter beim

Kaninchen. 96. Hypophysisgeschwulst. 101. Hypotonie. 305. Hysterl·sche Erblindung. 331.

I.

Iridocyklitis, rezidivierende, eitrige.

118. Iris, ÍStruktur der Vorderschicht der.

303.

- $\quad$ Anomalien der. 91.

K.

Kalkablagerung in den Ziliarfort-

sätzeđ. 219. , Kammerbucht, Anatomie der. 303. Kamnierwasser, Untersuchungen

des. 306. Keilbein, Röntgenbefunde im Be-

reiche des bei Sehstörungen. 335. Keratitisparenchymatosa trau-

matica. 168. Keratokonus. 323.

Korrektion des, 328. Keratoplastik. 322. -Kolo borne. 94.

Vererbung der. 317.

am Sehnerveneintrítt. 51. Konvefgenz, Einfluß der auí die

Verengerung der Pupille. 329. Kopfschüsse, Veränderungen in der

Augenhöble bei. 115.

Kriegsblin dheit. 317. ':';

Kurzsichtigkeit, Probleme der

Vererbung und der Erwerbu $\cdot n g$ døt

262.

- $\quad$ hochgradige und Glaukom. 308.';

$\mathrm{L} \cdot$

Lichen scrofulosorum der Binde-

haut. 222. Licht, Wirkungen des bei toxischen

Amblyopien. 32ã. Lichtsinn, Fehlerquelle bei der

Untersuchung des. 231. Lidschlußreaktion dérPupille,

315. . -,-. -·.•"..',';

354 Sach-Register für Band XLIV.

Linse, angeborene Verschiebung der I beiden durcbsichtigen. 104.

- $\quad$ Augenentzündung durch Lösung

von Linsenmassen in die Augen- 
flüssígkeit. 99. Linseneiweiß, ßausteíne des. 318. Linsenluxation, Verhalten der Zo-

nula bei Spontan luxation in die

Vorderkammer. 96. Liquor und Sehnervenbefund beiSy-

phylis. 312. Literaturberichte:

Deutsche L. 90.

HolГåndische. 96. Literaturverzeichnisse: 118, 233,

345. Lymphangi oma cysticum der Orbita. C5.

JT.

Macula lutea, Lage der in Bezug auf den Sehnerven. 98.

- $\quad$ Heterotopie der. 92.Makropsie, Genese der akkommo-

dativen- 13i. Megalo cornea. 93. Melanom der Aderhaut. 326. Mikro cornea. 94.

Mikrophthalmus, einseitiger. 94. Mikropsie, Genese der akkommo-

dativen. 17/82. Milchin j ektionen, parenterale. 145,

178, 184, 216. Miliartuberkulose, akut $\theta$ durch

traumatische Ruptur einer tuber-

kulösen Niere. 338. Mitbewegung. 101. Mißbildungen. $90 \mathrm{ff}$.

- $\quad$ Sehsphäre bei. 312.Myotica, Schädigung der normalen

Fovea durch. 341.

N. Nachtblindheit, idíopathische mit

Xerophthalmie. 100. Naevus der Lider. 102. Nase und Auge. 311. Nasenhöhle, Verhältnis der

Neuritis

retrobulbaris zur. 191, 194, 196 ff. Nebenhöhlenentzündung, Au-

genkomplikationen bei. 100. Nernstspaltlampe. 333. Nervusabducens, Abducensbrücke.

95. Netzhautablösung, Spaltungsver-

minderung bei. 318. Neuritis retrobulbaris, Häufung

der gegenüber der Zeit vor dem

Kriege. 38, 105, 106, 198.

- Verhältnis der zur Nasenhöhle. 191,

194, $196 \mathrm{ff}$.

- $\quad$ und multiple Sklerose. 125.

Novokain, Vergiftungserscheinungen in Form epileptischer Krämpíe bei Anwendung von N. zur Leitungs-unempfindlichkeit. 250.

Nystagmus, Zusammenhang zwi-schen der Richtung des und dem Reize in einem Bogengange. 99.

0.

Ophthalmia sympathica und Ge-

hörstörungen. 337. Optochinbehandlung. 313. Orbita, röntgenologiscbe DarstelluDg der. 334.

P.

Papilla nervi oi $3 / 4$ tici, Beziehungen der Augenbecherspalte zur. 316.

Papillitis, ülaukom nach. 308.

Perimetrie mit physiologischen Гarbenobjekten. 309.

Periphlebitis retinae tuber-c u 1 o s a. 324 .

Phlyktäne. 100.

- $\quad$ spezifische antituberkulöse Behand-

lung der. 319, 339. Pityriasis lichenoides chronic a der Lider und der Kon- 
junktiva. 2." > 3. Polykorie. 91. Proteinkörpertherapie. 145,178,

184, 216. Pupille, Einfluß der Konvergenz

und Akkommodation auf die Ver-

engerung der. 329. Pupilienreaktion, hemianopische.

315. Pupillenstarre, reflektoris che

durch Grippe bedingt. 44. Pupillenweite, Einfluß der auf den

Augendruck bei Glaukom. 325.

R.

Rheostat in Schnurform zum An-schluß an den Simonschen Augen-spiegel. 328.

Richtungslokalisation, ein neues Gesetz der. 311.

Röntgenologische Darstellung der Orbita. 334.

Röntgenstrahlen, Schädigungen am vorderen Augenabschnitt durch R. an einem wegen

Aderhaut-sarkom be $1 / 8$ trahlten Bulbus. 160.

Rot-Grünbíindheit. 310.

S. Sarkom, Entstehung des traumati-schen. 338.

metastatisches am Auge. 100. Scherenbrillen. 326. Sehnerv, Heterotopie des. 92.

senile Veränderungen am. 314.

Namen-Register fur Band XLIV.

355

Sehnerveneintritt, Kolobom am.

51. Sehprüíung, Eínfluß psycbischer

i'aktoren bei der S. kleiner Kinder.

328. Sehschärf enbestimmung. 320. Sehsphäre bei Mißbildungen des

Auges. 312. Sekretionsvorgänge, physiolo-

gische im Auge. 305. Sklerae, blaue bei Fragilitas ossium.

100. Sklerose, multiple und Neuritis retro-

bulbaris. 125. Skroíulöse Augenleiden, Be-

handlung der mit ultravioletten

Strahlen. 117. Spaltlampenmikroskop, Diagnose

partieller und totaler Vorder-

kammeraufhebunginittels des. 23?. Spaltungsverminderung bei Netz-

hautablösung. 318. Star, doppelseitiger totaler mit Quel-

lungsglaukom nach elektrischem

Schlag. 323.

- $\quad$ und Syphilis. 100.Syphilis, Liquor und Sehnerven-

befund bei. 312 .

- $\quad$ und Katarakt. 100.

T.

ïrachom. 99.

Tränendrüsen, hereditäre Ptosis der

orbitalen. 95. Tränenkanal, Druck beim üurch-

spritzen des. 101. T r ä n e n w e g e, familiäres Verkommen

resp. Vererbung von Erkrankungen

der. 257.

Tuberkulide der Conjunctiva *"• Tuberkulose und Trauma, $11^{\wedge}$. 
- $\quad$ speziñsche Diagnostik und TheГaPie

der. 232 (Bz.), 319, 339.

ü.

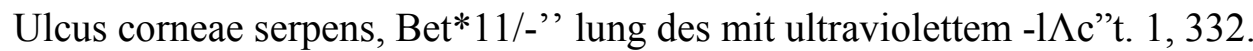

Ultraviolette Strahlen. 31":

bei skrofulösen Augenleiden. *

Behandlung entzündlicher Jíorn-

hauterkraDkungen mit. $1, \delta \check{\partial}_{i}$.

ünfallkunáe. 113, 336.

Unf allneurose. 115.

Unterschiedsempfin dliehkeJl, t' motorische und optische bei erkrankungen des Sehorgans. “ $\mathrm{V}$.

Verschüttungen, pathology0 eAnatomie der. 113.

Versicherungskunde. $113, \mathrm{i}^{3} / \mathrm{s}^{\wedge} \mathrm{V}$,

Vorderkammer, Ersatz der im mensehliehen Auge. 304.

Vorderkammerauf hebung, Vie ', gnose partieller und totaler mi** s Spaltlampenmikroskop. 287.

$\mathrm{X}$.

Xerophthalmie bei idiopathisCneГ Hemeralopie. 100.

Z.

Ziliarkörper, B,ingknoten des Erythema nodosum. 231.

Zonula, Verhalten der bei Spontan- luxation der Linse in die Vor ̋̂̃er" kammer. 96.

Zyklodialyse. 225.

Namen-Register für Banð XLIV.

Die fett gedruckten Zahlen bezeichnen Original-Artikel.

Adam 321, 328. Ascher 307. Axenfeld 308, 324.

B.

Bachstez 38,110, 188,

215, 220. Bandelier 232. Becker, H. 323. Behr 315. Bei'gmeister 91.

Brandt 309. Burger 113. Birch-Hirschf eld 1,

330, 332. Bolten 100 .

C.

Carsten 91. Clausen 323. Comberg 13, 322. Cords 232, 308.

1).

Des ax 342. ten Doesschate y ' 100 .

E.

v.E. Eicken 326. Elschnig 322. Engelking 223. 30^. Esser 132. Eunicke 338. 